\title{
Modelo multicritérios para o controle de qualidade da fralda geriátrica para o uso hospitalar
}

Multicriteria model for the quality control of geriatric diapers for hospital use Modelo multicriterio para el control de calidad del pañal geriátrico para uso hospitalário

Recebido: 23/05/2020 | Revisado: 26/05/2020 | Aceito: 27/05/2020 | Publicado: 11/06/2020

Alexandra Oliveira de Matias Ferreira

ORCID: https://orcid.org/0000-0003-1003-2754

Universidade Federal Fluminense, Brasil

E-mail: alexandrauff@gmail.com

Zenith Rosa Silvino

ORCID: https://orcid.org/0000-0002-2848-9747

Universidade Federal Fluminense, Brasil

E-mail: zenithrosa@id.uff.br

Carlos Marcelo Balbino

ORCID: https://orcid.org/0000-0003-0763-3620

Universidade Federal Fluminense, Brasil

E-mail: carlosmbalbino@hotmail.com

Marina Izu

ORCID: https://orcid.org/0000-0002-9615-8341

Universidade Federal Fluminense, Brasil

E-mail: izu.marina@gmail.com

Cláudio José de Souza

ORCID: https://orcid.org/0000-0001-7866-039X

Universidade Federal Fluminense, Brasil

E-mail: claudioenfo@gmail.com

Daniela de Oliveira Matias

ORCID: https://orcid.org/0000-0003-4177-6799

Universidade Federal do Estado do Rio de Janeiro, Brasil

E-mail: danielamatiasenf@hotmail.com 


\section{Resumo}

O presente estudo tem o objetivo de levantar as possíveis fragilidades das especificações das fraldas geriátricas a fim de discriminá-las em aprovada; aprovada com limitações e reprovada pela probabilidade de risco de incidência e prevalência de lesões de pele por pressão e/ou umidade, segundo o pressuposto de que a qualidade dos componentes das fraldas geriátricas pode ter um papel importante no surgimento das lesões de pele. Empregou-se relato de experiência, com amostra de quatro fraldas geriátricas, de uma mesma embalagem. Foram usados para testes: azul de metileno, água potável e cálice graduado. Criadas escalas para medição de volume miccional e modelo multicritérios para a qualidade das fraldas geriátricas para uso hospitalar. Com a metodologia de testes de produtos industriais e Multi Criteria Decision Aid (MCDA) foi possível avaliar as fraldas geriátricas segundo a presença de níveis variados de umidade, após a aplicação de solução-teste de simulação de volumes miccionais. Entretanto, mesmo sem o peso sobre as fraldas geriátricas ou com aplicação de apenas 1/4 do peso especificado na embalagem, a cobertura ao toque com volume a partir de $400 \mathrm{ml}$, tanto a cobertura e o lençol, estavam com níveis diferentes de presença de umidade. Assim, existe a probabilidade da associação da qualidade das fraldas geriátricas com a incidência e prevalência de lesão por pressão e/ou umidade.

Palavras-chave: Controle de qualidade; Pesquisa operacional; Economia da Enfermagem; Pesquisa em administração de Enfermagem.

\section{Abstract}

The present study aims to raise the possible weaknesses of the specifications of geriatric diapers in order to discriminate them in approved; approved with limitations and disapproved by the probability of risk of incidence and prevalence of skin lesions by pressure and/or moisture, according to the assumption that the quality of the components of geriatric diapers may play an important role in the appearance of skin lesions. We used an experience report, with a sample of four geriatric diapers, of the same package. They were used for testing: methylene blue, drinking water and graduated chalice. Scales were created for micition volume measurement and multicriteria model for the quality of geriatric diapers for hospital use. With the methodology of testing of industrial products and Multi Criteria Decision Aid (MCDA) it was possible to evaluate geriatric diapers according to the presence of varied levels of moisture, after the application of a test solution for simulation of voiding volumes. However, even without the weight on the geriatric diapers or with the application of only $1 / 4$ of the weight specified in the package, the touch cover with volume from $400 \mathrm{ml}$, both the 
cover and the sheet, had different levels of presence of moisture. Thus, there is a probability of the association of the quality of geriatric diapers with the incidence and prevalence of pressure and/or moisture injury.

Keywords: Quality control; Operations research; Economics, Nursing; Nursing administration research.

\section{Resumen}

El presente estudio tiene como objetivo plantear las posibles debilidades de las especificaciones de los pañales geriátricos con el fin de discriminarlos en aprobados; aprobado con limitaciones y desaprobado por la probabilidad de riesgo de incidencia y prevalencia de lesiones cutáneas por presión y/o humedad, según el supuesto de que la calidad de los componentes de los pañales geriátricos puede desempeñar un papel importante en la aparición de lesiones cutáneas. Usamos un informe de experiencia, con una muestra de cuatro pañales geriátricos, del mismo paquete. Se utilizaron para las pruebas: azul de metileno, agua potable y cáliz graduado. Se crearon básculas para la medición del volumen de micition y modelo multicriterio para la calidad de los pañales geriátricos para uso hospitalario. Con la metodología de ensayo de productos industriales y Multi Criteria Decision Aid (MCDA) fue posible evaluar pañales geriátricos de acuerdo con la presencia de diversos niveles de humedad, después de la aplicación de una solución de prueba para la simulación de volúmenes de vaciado. Sin embargo, incluso sin el peso en los pañales geriátricos o con la aplicación de sólo 1/4 del peso especificado en el envase, la cubierta táctil con volumen de $400 \mathrm{ml}$, tanto la cubierta como la sábana, tenía diferentes niveles de presencia de humedad. Por lo tanto, existe una probabilidad de asociación de la calidad de los pañales geriátricos con la incidencia y prevalencia de lesiones por presión y/o humedad.

Palabras-clave: Control de calidad; Investigación operativa; Economía de la Enfermería; Investigación en administración de Enfermería.

\section{Introdução}

As mudanças atuais nos sistemas de saúde são pautadas na escassez de recursos em saúde e isso pode impactar diretamente na decisão pela compra de produtos pelos gerentes de recursos materiais de um hospital (Porzsolt, 2015; Castilho, \& Gonçalves, 2010). Neste sentido, muitas empresas, preocupadas em garantir a qualidade de seus produtos e serviços, 
passaram a utilizar cada vez mais as ferramentas do controle estatístico da qualidade (Ramos, Almeida \& Araújo, 2013).

A preocupação com as características da qualidade de uma empresa de telefonia motivou o físico, engenheiro e estatístico Walter Andrew Shewhart a desenvolver uma série de estudos com diversas ferramentas de controle em 1924, o que lhe rendeu o título de "pai do controle estatístico de processo" (Ramos, Almeida \& Araújo, 2013). Quase 100 anos passados das suas investidas de grande sucesso nas empresas, o conceito de qualidade reflete atualmente uma perspectiva do mercado levando a um cenário em que os consumidores se tornam mais exigentes quanto aos produtos e serviços que lhes são oferecidos (Ramos, et al., 2013; Carpinetti, 2012).

O gerenciamento de materiais nas organizações compreende o processo gerencial para aquisição e disponibilização de materiais já manufaturados, essenciais para a produção de serviços de saúde. A ineficácia nessa área propicia processos de trabalhos com materiais de baixa qualidade, em decorrência da falta de parâmetros de qualidade que se configura um desafio gerencial para todos os gestores de saúde que precisam garantir a qualidade dos cuidados de saúde (Castilho \& Gonçalves, 2010).

Um produto bastante empregado em ambiente hospitalar são as fraldas geriátricas, como compreendemos hoje, surgiram no final da década de 90, do século passado, a partir da criação de materiais compostos por polímero superabsorvente (PSA). São redes hidrofílicas que podem absorver e reter grandes quantidades de água ou soluções aquosas. Elas podem absorver água em até $100.000 \%$.

Os PSA comuns são geralmente materiais higroscópicos semelhantes ao açúcar branco, usados principalmente em fraldas descartáveis e outras aplicações, incluindo no uso agrícola (Lejcuś, Śpitalniak, \& Dąbrowska, 2018; Zohuriaan-Mehr, \& Kabiri 2008). Sendo assim, a fralda é um produto composto por diversos componentes químicos com função de absorção, barreira e proteção da pele, usada nos casos em que o paciente tenha o risco de ficar exposto por longo tempo a urina e fezes em decorrência da perda da capacidade coordenada de armazenamento e eliminação de urina (Lejcuś, Śpitalniak, \& Dąbrowska, 2018; Locks, \& Santos, 2016).

O conceito de fralda está inserido na dimensão da qualidade do produto, que passou por mudanças drásticas nos seus atributos para se adequar ao uso de um determinado mercado. No passado, as fraldas de fibra natural tinham a especificação de absorção pouco desenvolvida, carecendo de outra cobertura, bem como era desprovida de especificações 
relacionadas à barreira. Além disso, tinham outras tecnologias associadas para fechar a fralda. Por causa disso, a fralda sempre foi um conceito multiatributos.

A qualidade do produto e a gestão de qualidade são as dimensões de qualidade mais relevantes e amplamente discutida na literatura (DeFEO \& Juran, 2015). No controle de qualidade são comparadas as dimensões previstas e aquela realmente alcançada. Assim, os produtos são considerados de qualidade quando se adequam aos padrões previamente definidos. Entretanto, quando os padrões, também chamados de especificações, não são previamente definidos, a qualidade torna-se ambígua e a aceitação ou rejeição do produto deve ser levada à discussão (Zohuriaan-Mehr \& Kabiri, 2008).

Portanto, na avaliação da qualidade de um produto para os cuidados de enfermagem deve ser levado em conta, dentre outros aspectos, os atributos intrínsecos e o seu significado para o cuidado de enfermagem pelos resultados esperados da sua aplicação na prática. Desse modo, a eficiência desta avaliação é algo que se relaciona em grande parte na genialidade dos profissionais que atuavam na padronização de materiais (Castilho, \& Gonçalves, 2010).

Um produto é considerado de qualidade quando atende exatamente aos padrões préestabelecidos e exibe as exatas especificações adotadas (DeFEO, \& Juran, 2015). Todavia, não existem estudos que fazem associação da ocorrência das lesões de pele pelo uso de fraldas geriátricas em diferentes grupos etários e volumes miccionais.

Tendo em vista que a aquisição de um produto de baixa qualidade irá interferir no planejamento e na execução das atividades assistenciais comprometendo, consequentemente, a auditoria dos recursos usados para a prestação de cuidados, podendo causar o retrabalho, aumentando o tempo de internação, danos aos pacientes e aumento dos custos por internação(Scholze et al., 2017).

Assim, a relevância deste estudo se baseia na necessidade de obter parâmetros mínimos de qualidade das fraldas geriátricas direcionados ao ambiente hospitalar para ser comparados por diferentes critérios. Isso é importante porque uma revisão de literatura mostrou que alguns cuidados preventivos com a pele, com estratégias de manejo intestinal para controlar a incontinência fecal e a luz polarizada aplicada uma vez por dia durante 10 minutos nas lesões na área sacral e do calcanhar não foram efetivos, mas a aplicação profilática de espuma de silicone se mostrou significativamente efetiva na diminuição na incidência de lesões por pressão sacral Mäki-Turja-Rostedt, Stolt, Leino-Kilpi, Haavisto, 2019). Desse modo, a cobertura da fralda geriátrica pode ser inefetiva como uma barreira para evitar que a urina e/ou as fezes entre em contato com a pele dos pacientes tendo relação com a formação da lesão de pele. 
A partir disso, supõe-se que existe uma dinâmica das fraldas geriátricas com o peso dos pacientes, saturação do polímero e da cobertura pela presença de diferentes volumes de urina e fezes que promovem padrões de atrito na pele, favorecendo ao surgimento das lesões por pressão, principalmente em indivíduos com mobilidade prejudicada (Pittman, Beeson, Dillon, Yang \& Cuddigan, 2019). Com base nisso, pressuposto de que a qualidade dos componentes das fraldas geriátricas pode ter um papel importante no surgimento das lesões de pele.

Estudos na área da qualidade de produtos hospitalares são necessários para compreender como diferentes especificações determinadas pelos fabricantes se comportam na vida real. Frente o exposto, este estudo tem como objetivo: levantar as possíveis fragilidades das especificações das fraldas geriátricas a fim de discriminá-las em aprovada; aprovada com limitações e reprovada pela probabilidade de risco de incidência e prevalência de lesões de pele por pressão e/ou umidade.

\section{Metodologia}

Para este estudo, os autores se propuseram realizar um estudo na modalidade relato de experiência, de cunho descritivo e qualitativo (Pereira et al., 2018). Empregando-se para os isso duas fases: o teste de produtos industriais e a estruturação de modelo de multicritérios construtivista (Back \& Leal, 1992; Longaraya \& Leonardo, 2014).

A amostra foi composta por 4 fraldas, do pacote de 8 fraldas geriátricas. $\mathrm{O}$ uso de amostra de lotes de produtos é uma técnica de amostragem usada no controle de qualidade de produtos, nos casos em que se deseja reduzir os custos com produtos de baixa qualidade com emprego de testes (Rosenfeld, 2013). O pacote de fraldas geriátricas foi disponibilizado pelo fabricante para testes em lócus, localizada na cidade de São Gonçalo, no Rio de Janeiro, Brasil. Os testes foram realizados em setembro de 2019.

O método escolhido foi de Back \& Leal (1992) para testes de produtos industriais, no qual eles estabelecem que a qualidade dos produtos se dá pelos diversos testes realizados em todas as fases do ciclo de vida do produto. Eles pontuam que os testes devem ser realizados em seis etapas: tipos de testes; planejamento do teste; preparação do teste; realização do teste; seleção do laboratório e dos testes independentes. 
Fase: teste de produtos industriais

\section{Tipos de testes}

Os testes foram elaborados para avaliar as especificações das fraldas geriátricas de uso hospitalar descritas na embalagem, segundo o fabricante:

1) Elásticos nas pernas: evitam vazamento e promovem segurança e conforto;

2) Formato anatômico desenhado para oferecer comodidade, eficiência e liberdade de movimento;

3) Cobertura filtrante: eficiência e proteção com suavidade e muito conforto;

4) Flocos de gel superabsorventes: excelente absorção de líquidos, menor umidade e conforto;

5) Indicador de umidade.

\section{Planejamento do teste}

A composição da fralda geriátrica, segundo a embalagem, era de fibras de celulose; fibra de prolipropileno; polímeros termoplásticos; polímero superabsorvente; fita reposicionável; filme de poliestileno e elástico, sem especificação das medidas usadas para confecção do produto. Foram cedidos no pacote a informação do tamanho G e GG. Na embalagem estavam descritos apenas dois atributos: tamanho da cintura, o peso e seus subatributos, a saber: tamanho médio direcionado a pessoas com pesos de 40 a $70 \mathrm{~kg}$ e cintura de até $120 \mathrm{~cm}$; tamanho grande para pessoas entre 70 a $90 \mathrm{~kg}$ e cintura de 120 a $150 \mathrm{~cm}$ e extragrande acima de $90 \mathrm{~kg}$ e cintura entre $150-160 \mathrm{~cm}$.

\section{Materiais usados nos testes da fralda geriátrica}

Foram usados uma garrafa de água mineral vazia, uma ampola de azul de metileno com 5 mililitros $(\mathrm{ml})$, um cálice graduado de plástico, 4 pacotes de gazes com 5 unidades, uma caixa de soro glicosado $5 \%$ de $500 \mathrm{ml}$, balança antropométrica Filizola ${ }^{\circledR}$ digital com capacidade para medir até 200 quilogramas $(\mathrm{Kg})$, uma maca e um lençol branco de algodão, o temporizador disponibilizado pelo Google ${ }^{\circledR}$ para smartfones e a câmera de celular com resolução de 12 mega pixels. 


\section{Preparação dos testes}

Como as fraldas determinam diferentes atributos pelos parâmetros muito amplos dispostos na embalagem, existe a incerteza da quantidade de volume que cada uma poderia suportar, uma vez que elas pareciam idênticas, podendo resultar em várias perspectivas possíveis para cada característica dos vários atributos. Para solucionar esse problema foi feita uma solução de azul de metileno diluído em $1500 \mathrm{ml}$ de água de torneira, que foi fracionada por meio da medição em um cálice graduado de plástico em 200 ml, 300 ml, 400 ml e 500 ml.

$\mathrm{Na}$ contraprova foram usados $100 \mathrm{ml}$ da primeira solução para nova diluição para $1400 \mathrm{ml}$, a fim de diminuir o tom de azul e evitar que novas e antigas manchas pudessem confundir a leitura do teste de extravasamento. Os volumes foram escolhidos para simular diferentes comportamentos miccionais de pacientes em ambiente hospitalar. As quatro fraldas eram do mesmo lote e tamanho, dispostas lado a lado sobre uma maca, identificadas com esparadrapo com os respectivos volumes a serem testados acima de cada uma.

\section{Descrição dos testes}

Os testes da qualidade das fraldas geriátricas foram realizados usando as especificações projetadas nas embalagens que foram divididos em teste de inspeção; teste de toque e teste de umidade que é subdividido em: teste de barreira; teste de peso sobre a fralda e teste de deformidade, como é descrito a seguir:

- Teste de inspeção foi realizado através da observação do brilho da cobertura da fralda geriátrica e a saturação do gel pelo lado externo após a aplicação da solução-teste;

- Teste do toque foi realizado por digito-pressão sobre as fraldas geriátricas e teve o intuito de encontrar pontos de umidades e deformidades das laterais na cobertura em todas as etapas dos testes;

- Teste de umidade foi dividido em teste de barreira e teste de peso sobre as fraldas geriátricas. No teste de barreira, elas foram dispostas sobre um lençol branco de uso hospitalar para detectar o extravasamento da solução-teste no lençol na aplicação imediata, após uma hora. Para testar a umidade associado ao peso, as fraldas geriátricas foram submetidas a um peso de aproximadamente $1 / 4(17,075 \mathrm{~kg})$ do peso mínimo determinado (70 kg) referido pelo fabricante. Para isso, usou-se uma caixa de ampolas de soro glicosado a $5 \%$ de $500 \mathrm{ml}$ com 30 unidades. A presença do extravasamento foi realizada com emprego de cinco folhas de gaze colocadas sob a 
(CC BY 4.0) | ISSN 2525-3409 | DOI: http://dx.doi.org/10.33448/rsd-v9i7.4835

cobertura da fralda geriátrica, simulando algo que poderia acontecer na pele. O tempo do peso sobre as fraldas foi de 5 minutos.

- Teste de deformidade: Após uma hora de despejada a solução-teste, as fraldas foram suspensas pelas abas das pontas superiores e inferiores para observar o peso no fundo e a perda da sua estrutura. Fraldas pesadas ficam com formato de "funil", com peso concentrado no fundo causando afrouxamento das bordas superiores e laterais.

\section{Realização do teste}

Dispensou-se em cada fralda geriátrica Figura 1 o volume previamente determinado no centro de cada uma, deixando-as abertas e expostas ao meio ambiente por período de uma hora. Depois da primeira hora, foram realizadas avaliações das fraldas pelos critérios de qualidade por meio dos testes de extravasamento, barreira, peso e teste de deformidade.

Figura 1. Aplicação da solução-teste nas fraldas geriátricas identificadas com volumes de $200 \mathrm{ml}, 300 \mathrm{ml}, 400 \mathrm{ml}$ e $500 \mathrm{ml}$.

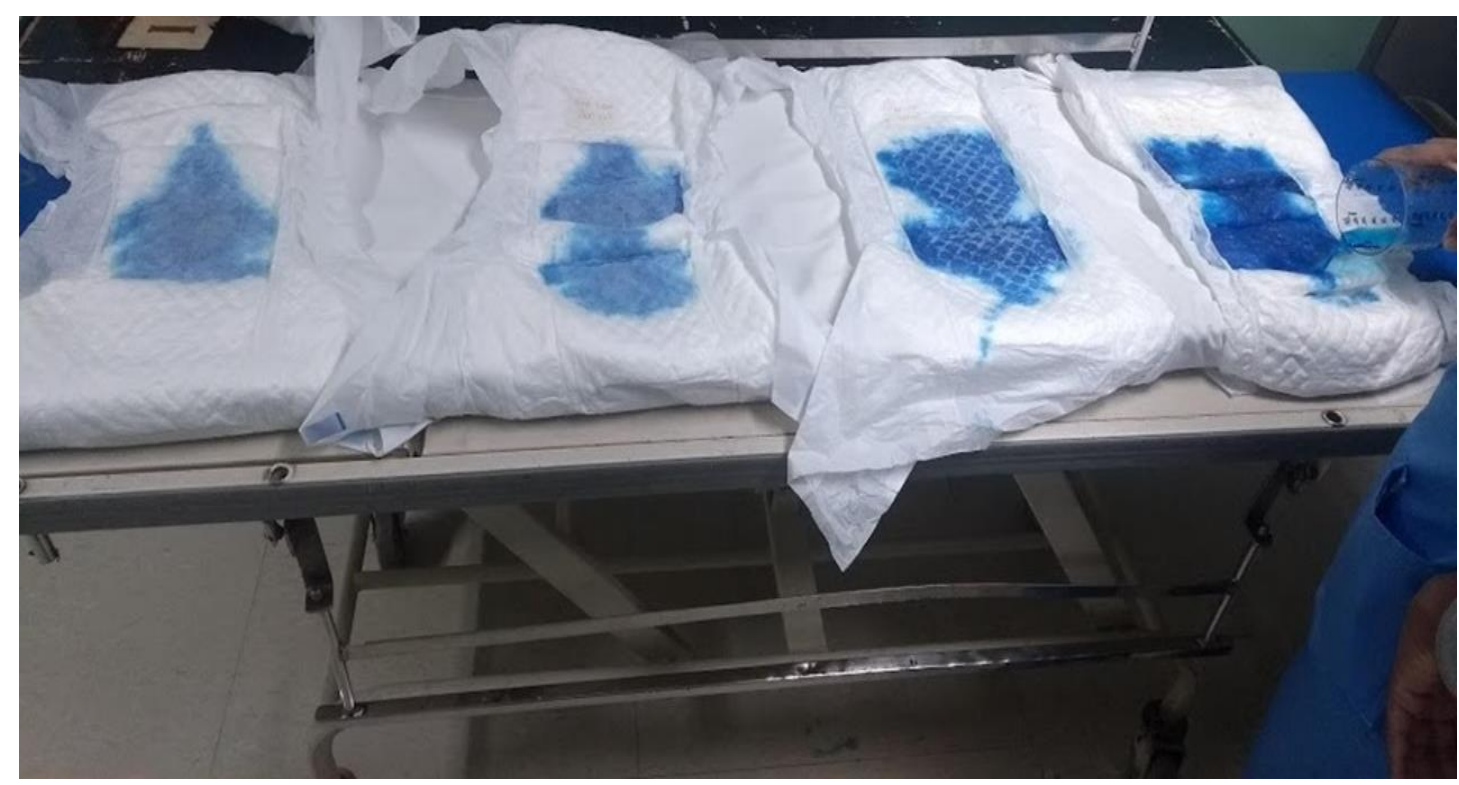

Fonte: Autores.

A Figura 1 mostra como as fraldas foram dispostas na maca para a aplicação da solução-teste. Iniciou-se pela fralda esquerda com $200 \mathrm{ml}$, seguindo-se até o final com volume de $500 \mathrm{ml}$.

\section{Seleção do laboratório e dos testes independentes}

O cenário foi o corredor de um hospital universitário do Rio de Janeiro. 


\section{Testes independentes}

Não foram realizados testes independentes.

\section{Fase: Multi Criteria Decision Aid (MCDA)}

O MCDA constitui-se em diversas abordagens metodologias de análise de decisão. Entre esses métodos se tem o construtivista que é constituído de três fases: estruturação, avaliação e recomendação (Longaraya \& Leonardo, 2014). A etapa de avaliação foi implicada tornar a leitura do modelo mais fácil para ser empregado por gestores de saúde nos hospitais, uma vez que a maioria deles não dominam os modelos matemáticos de decisão.

Mediante a essa avaliação foi usada uma escala de recomendação a fim de discriminála em aprovada; aprovada com limitações e reprovada pela probabilidade de risco de incidência e prevalência de lesões de pele.

Os 8 critérios usados para avaliação da umidade na cobertura da fralda geriátrica e classificação em ordem alfabética para melhor visualização nos resultados foram: a) inspeção; b) umidade imediata; c) umidade sob pressão; d) peso; e) toque; f) cobertura brilhante; g) extravasamento para o lençol e h) presença de deformidade.

\section{Critério considerado crítico}

O critério considerado crítico pode ser usado como uma regra empregada por avaliadores para decidirem se recomendam ou não a compra das fraldas geriátricas por faixa etária, volume miccional e peso para uso hospitalar.

A umidade, presente em qualquer teste, em qualquer quantidade, estará em desacordo com as especificações descritas pelos projetistas, algo que determina a reprovação do uso das fraldas geriátricas para o uso hospitalar, mesmo que o indicador de umidade não se modifique com a presença de urina e/ou fezes.

Se a umidade estiver presente, a aquisição pode ser embargada pelo gestor de recursos materiais por se tratar de um produto considerado de baixa qualidade, pela probabilidade de alta incidência e prevalência de lesões de pele.

Escala miccional

A escala classificatória de volume miccional é uma medida ordinal da micção normal masculina e feminina para as várias faixas etárias. Por exemplo: a fralda classificada em 1 gota suporta apenas uma micção de $100 \mathrm{ml}$ ou duas micções de $200 \mathrm{ml}$ e assim por diante. 
Foi escolhida a gota como símbolo da capacidade de absorção do PSA e também por ser usada comercialmente em absorvente de uso feminino para se referir a capacidade absortiva dos polímeros em produtos usados para higiene e prevenção e tratamento de lesões.

Usualmente, os produtos são escalonados em três gotas, mas para esse estudo, optouse em usar cinco níveis para discriminar melhor os volumes possíveis dos adultos. Na Figura 2 faz menção a categorização das gotas por intervalo de volume miccional.

Figura 2. Escala de volume miccional.

$100 \mathrm{ml}-199 \mathrm{ml}$
$200 \mathrm{ml}-299 \mathrm{ml}$
$300 \mathrm{ml}-399 \mathrm{ml}$
$400 \mathrm{ml}-499 \mathrm{ml}$

Fonte: Autores.

Modelo multicritérios de avaliação da qualidade da fralda geriátrica para uso hospitalar

Um modelo multicritério faz parte da fase de planejamento do gerenciamento de recursos materiais empregado para avaliar as diferentes especificações de um produto e seu desempenho na vida real antes do processo de compra. Para que os gestores em hospitais tomem a decisão acerca da fralda geriátrica que melhor se adeque as necessidades dos doentes sob sua responsabilidade.

Elaborou-se uma escala de apoio à tomada de decisão acerca da qualidade das fraldas geriátricas, segundo alguns critérios inferidos dos testes realizados. Essa modelo foi pensada como uma ferramenta estratégica para apoiar o julgamento e tomadas de decisão daqueles que estão à frente das comissões de padronização de materiais.

Após realização dos testes, são três possíveis decisões: aprovada, aprovada com limitações e reprovada. Aprovada: após os testes, a fralda atendeu a todos os critérios de qualidade estabelecidos pelos projetistas; aprovada com limitações: critérios de qualidade considerados aceitáveis, mas não ideais e reprovada: totalmente fora dos padrões determinados pelo hospital. Após os testes, caberá aos decisores tomar as decisões segundo critérios como preço e dimensionamento da equipe de enfermagem. 


\section{Coleta e análise de dados}

A coleta de dados realizada por meio de formulário com perguntas elaboradas a partir do critério avaliativo, projetado com base nas informações da embalagem das fraldas geriátricas, com respostas dispostos de forma dicotômica (sim ou não), as quais foram: a) presença de marcas de umidade do lençol; b) presença de formato funil; c) presença de umidade ao toque; d) presença de umidade ao peso; e) presença de umidade espontânea. A análise estatística descritiva se deu apenas pela frequência absoluta.

\section{Resultados}

Na Figura 3 estão dispostos os diferentes padrões de umidade com o emprego de cinco folhas de gaze, há presença de umidade pelo aspecto brilhoso da cobertura e manchas de extravasamento no lençol, evidenciando que a cobertura pode transferir umidade para pele, inclusive molhando os lençóis que cobrem os leitos.

Figura 3. Resultados dos testes nas fraldas geriátricas.

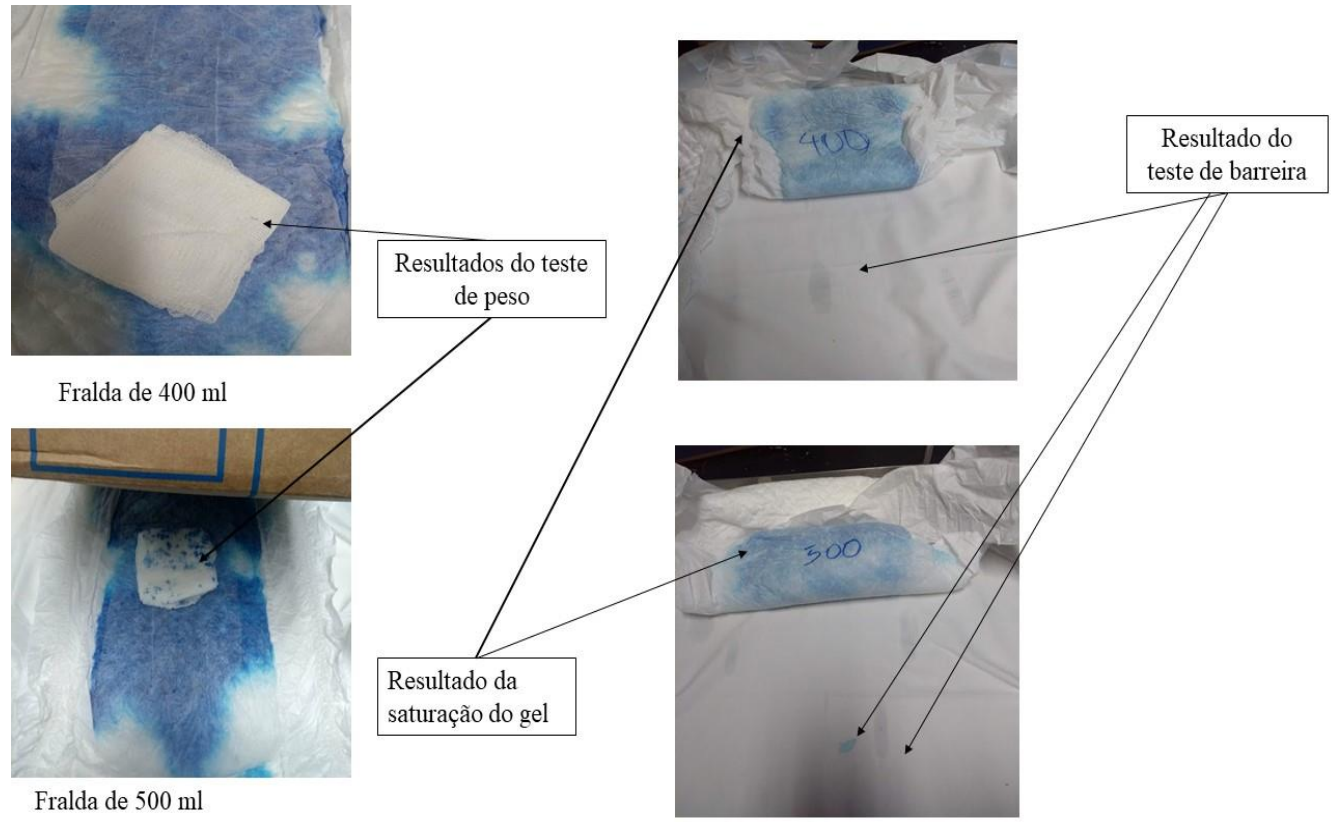

Fonte: Autores.

Os resultados do teste de umidade após despejar os volumes da solução-teste. Logo após 5 minutos, na inspeção e toque com $200 \mathrm{ml}$ e $300 \mathrm{ml}$, a sensação era cobertura seca e o teste de extravasamento para o lençol foi negativo. Ambas apresentaram extravasamento para o lençol, sendo que a fralda com $500 \mathrm{ml}$ apresentou mais pontos molhados do que a fralda 
(CC BY 4.0) | ISSN 2525-3409 | DOI: http://dx.doi.org/10.33448/rsd-v9i7.4835

com $400 \mathrm{ml}$. Também se evidenciou que as fraldas geriátricas com volume de 400 e $500 \mathrm{ml}$, após 5 minutos, na inspeção e ao toque, as coberturas davam a sensação de superfícies molhadas, sendo que a fralda geriátrica identificada com $500 \mathrm{ml}$, após o toque dos dedos, observou-se escoamento da solução-teste entre eles. Na Figura 4 estão dispostas as fraldas geriátricas após 5 minutos de aplicação da solução-teste.

Figura 4. Comparação das coberturas das fraldas geriátricas.

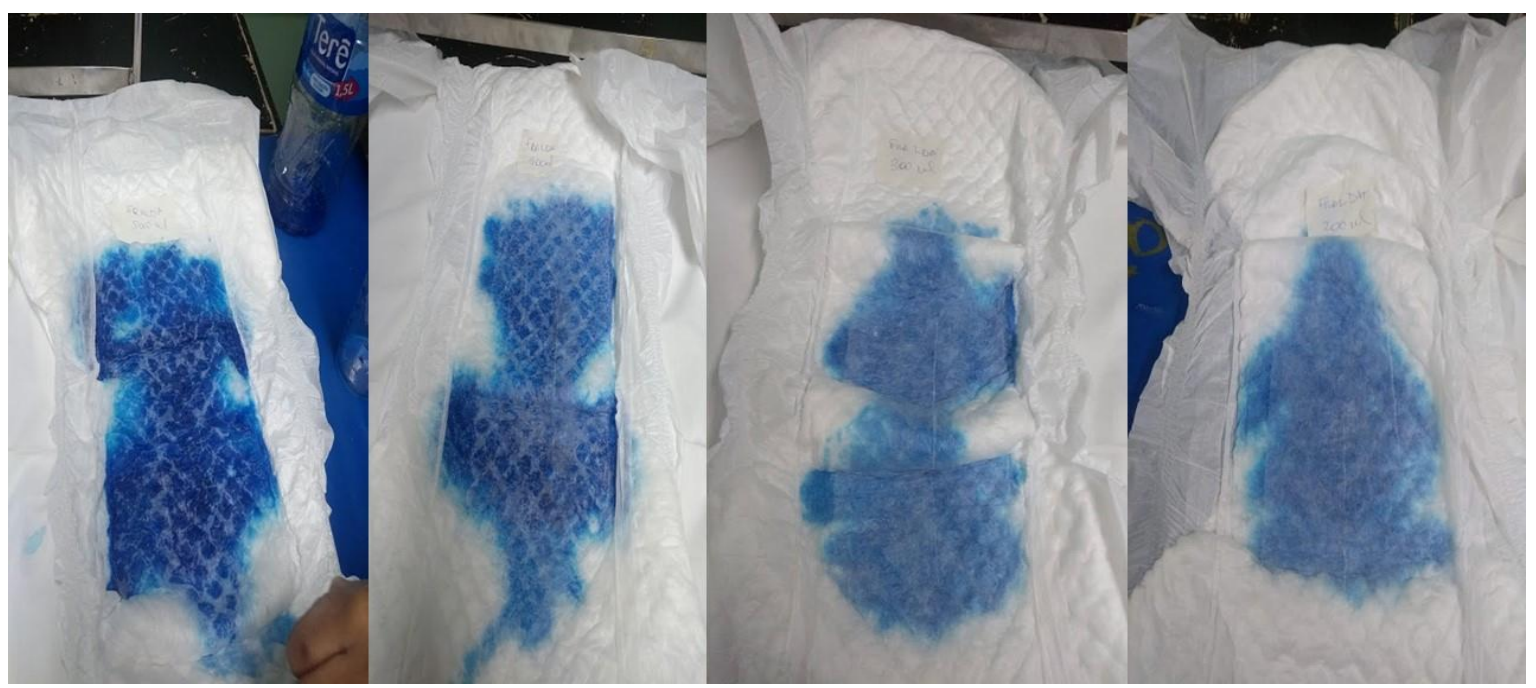

Fonte: Autores.

As fraldas estão dispostas de maior volume $500 \mathrm{ml}$ até o de menor volume $200 \mathrm{ml}$, da direita para esquerda. Sendo assim é possível constatar na fralda geriátrica de $500 \mathrm{ml}$ cor brilhante em toda a cobertura da fralda com $500 \mathrm{ml}$ e presença de pontos mais concentrados se comparado aquela de $400 \mathrm{ml}$, após 5 minutos depois. No teste de peso, as fraldas de $400 \mathrm{ml}$ e $500 \mathrm{ml}$ tinham aparência de funil quando suspensas pelas abas. Com base nos testes levantados, no Quadro 1, nota-se que é possível usá-los na prática para modelagem de diferentes critérios para avaliação da qualidade da fralda geriátrica para o uso hospital. 
(CC BY 4.0) | ISSN 2525-3409 | DOI: http://dx.doi.org/10.33448/rsd-v9i7.4835

Quadro 1. Modelo multicritérios para a qualidade das fraldas geriátricas para uso hospitalar.

\begin{tabular}{|l|l|l|l|l|l|l|l|l|}
\hline Especificações & \multicolumn{7}{|c|}{ Critérios } & Avaliação \\
\hline $\begin{array}{l}\text { Volumes da } \\
\text { solução-teste }\end{array}$ & A & B & C & D & E & F & G & Recomendação \\
\hline $200 \mathrm{ml}$ & Não & Não & Não & Não & Não & Não & Não & Aprovada com limitações \\
\hline $300 \mathrm{ml}$ & Não & Não & Não & Não & Não & Não & Não & Aprovada com limitações \\
\hline $400 \mathrm{ml}$ & Sim & Sim & Sim & Sim & Sim & Sim & Sim & Não aprovada \\
\hline $500 \mathrm{ml}$ & Sim & Sim & Sim & Sim & Sim & Sim & Sim & Não aprovada \\
\hline
\end{tabular}

Fonte: Autores.

No Quadro 1, as fraldas do lote analisado apresentam bom desempenho com a aplicação de $200 \mathrm{ml}$ e $300 \mathrm{ml}$ de solução-teste e com peso 1/4 do especificado pelo fabricante. Sendo assim, as fraldas geriátricas numa mesma embalagem, foram aprovadas com limitações para uso na prática em hospitais na faixa miccional de 1 a 3 gotas.

\section{Discussão}

O projetista das fraldas geriátricas atuais, dentre as várias especificações, determinou que o peso e o tamanho da cintura dos pacientes são importantes para as comparações entre os produtos. Entretanto, neste estudo preliminar, parece as especificações não são adequados aos significados dos gestores de cuidados, no contexto de cuidados hospitalares, que têm a preocupação com as sérias consequências não intencionais e adversas com a umidade direta na pele em condições de racionamento de enfermagem (Mäki-Turja-Rostedt, 2018; Jones, Hamilton \& Murry 2015).

Autores argumentam que mentes criativas devem ser preocupar em desenvolver tecnologias que aprimorem o cuidado com vistas à qualidade assistencial (Balbino et al., 2020). Todavia, os riscos da incorporação tecnológica sem o devido controle de qualidade podem resultar em possíveis iatrogenias ainda mais que não se sabe se existem testes realizados em lócus com lotes de fraldas geriátricas antes da sua compra, o que torna a comparação da qualidade das tecnologias para o cuidado em saúde difícil de ser realizada.

A forma não controlada da aquisição das fraldas geriátricas por volume de micção, peso e idades diferentes pode favorecer a compra de produtos de baixa qualidade, que ficará em uso por longo tempo pelos pacientes, expondo-os a umidades, fricção e odores (Pittman, 
et al., 2019; Mäki-Turja-Rostedt, 2018). Embora, hoje, esteja claro a existência da ocorrência de lesões por pressão inevitável, em alguns casos, sugere-se uma possível etiologia da insuficiência cutânea que pode piorar com fraldas geriátricas de baixa qualidade (Pittman, et al. 2019).

A falta da composição dos itens da fralda geriátrica e a capacidade total absortiva descrita nas embalagens impedem comparações das medidas de qualidade em diferentes produtos. Os responsáveis pelo gerenciamento de materiais devem ter total atenção às definiç̧ões dos produtos para o uso na prática, com explicação dos parâmetros exatos para mensuração e associação dos efeitos capazes de minimizar o impacto das variabilidades na qualidade dos cuidados em saúde.

Adequação ao uso é um conceito desenvolvido por Juran que se refere a uma relação entre consumidor/clientes onde ele é o rei e os produtores produzem com base em determinadas conformidades pelas especificações determinadas (DeFEO \& Juran, 2015). Sabe-se que a competição global, bem como as exigências cada vez maiores dos consumidores, leva as organizações a procurar constantemente formas melhorar as especificações dos produtos e serviços com custos mais baixos, mas com elevada qualidade (Rosenfeld, 2013). Contudo, falhas na produção das fraldas geriátrica podem ter ocorrido e que não foram detectadas pelos fabricantes.

Como forma de examinar as relações entre o PSA, a cobertura e o peso, concentrou-se nos testes das especificações atribuídas pelos projetistas para que os resultados pudessem ser observados diretamente pelos gestores. Neste ponto, os resultados dos testes e as suas consequências para o conforto dos doentes pelo uso de um produto de saúde de qualidade é uma busca pela compreensão das relações que podem ser observados na prática.

No que tange aos doentes críticos o uso das fraldas geriátricas, elas podem causar diversas consequências adversas devido à instabilidade hemodinâmica dos pacientes, pode determinar outros tipos de resultados (Pittman, et al., 2019). Inclusive, os indicadores de qualidade da assistência de enfermagem relacionada à pele devem ser melhores analisados, pois já se sabe que existe o sinal de falha cutânea que mostra a presença do dano que ocorre na pele e nos tecidos subjacentes no final da vida, no ambiente de terapia intensiva, que não devem se associar a qualidade das fraldas geriátricas neste ambiente (Mani \& Ibrahim, 2017). Assim, não se pode decidir com certeza qual tipo de material é de qualidade numa organização contemporânea sem um entendimento genuíno de como os produtos interferem no processo clínico dos doentes. 
As categorias da fralda geriátrica que foram determinadas pelo projetista por uma escala ordinal de tamanho em médio, grande e extragrande, é uma forma de discriminar os grupos para qual cada tamanho é indicado, com a finalidade de minimizar o efeito das variabilidades dos pesos sobre a tecnologia analisada. As escalas ordinais são úteis, pois é uma forma simples para estabelecer uma classificação, na qual este número representa uma determinada categoria de uma variável (Costa, 2011). Entretanto, é uma escala de apelo conceitual em que a aferição observada é comparada com certos padrões aceitos.

O maior uso de PSA é em produtos de higiene pessoal, usados principalmente como absorventes para água e soluções aquosas para fraldas, produtos para incontinência adulta, produtos de higiene feminina e aplicações similares como substituição de materiais absorventes tradicionais, como tecidos, algodão, algodão e fibras de celulose (Zohuriaan, \& Kabiri, 2008; Marconato \& Franchetti, 2002). Eles são empregados nas fraldas por possuir boa permeabilidade, forte capacidade de absorção e capacidade de manutenção, sem nenhum fenômeno de infiltração de retorno sob uma certa pressão. Apesar de tantos benefícios, em certas doenças renais ou gastrointestinais que levam ao aumento de eliminações fisiológicas, os padrões aceitos de normalidade determinados pelo projetista com base em parâmetros de normalidade em ambientes domiciliares, podem ser considerados anormais para o emprego no mesmo grupo na hospitalização (Riella, 2018).

Como já foi dito, as fraldas geriátricas são compostas por diferentes componentes químicos. A parte mediana é formada por polímeros que são macromoléculas de alta massa molar, formadas por unidades de moléculas menores, chamadas monômeros (Lejcuś, et al. 2018). Estes reagem por adição ou condensação, mudando de aspecto quando hidratados sofrendo variação na absorção, dependentes de fatores ambientes e químico-físicos (Zohuriaan-Mehr \& Kabiri, 2017). A influência destes fatores nos resultados dos testes deve ser prevista nos testes de confiabilidade com água de torneira, que sem dúvida, não apresenta as mesmas características químicas da urina e nem das fezes humanas, o que pode mudar o formato final do PSA, podendo diferir em novos testes na prática.

O peso é o fator que pode contribuir para o desprendimento do líquido do polímero, aumentando a probabilidade de expor a pele da região sacral à umidade, principalmente porque as fraldas são indicadas para pacientes com pesos que podem variar em até 20 quilos (kg), 70-90kg, num mesmo modelo. Essa variação de peso é enorme para se ter a mesma quantidade de polímero e tipo de cobertura, uma vez que a variação do peso, determina também a variação da intensidade da força exercida sob o objeto. 
Uma pessoa saudável tem micções a cada 4-6 horas durante o dia, normalmente não tem micções no período noturno (Riela, 2018). Assim, as fraldas geriátricas podem ser diferentes pelo período diurno e noturno, sendo mais absortivas e mais resistentes para período noturno para não interferir no padrão de sono, como também as fraldas devem ser estratificadas pela quantidade de fezes ou urina que cada unidade pode suportar por cada uso, para que os profissionais ou famílias possam controlar e prever as trocas.

Durante o ciclo de vida de um produto, a falta de informações relevantes sobre eventos previstos, a existência de problemas tecno-econômicos cujos procedimentos de solução são desconhecidos, assim como, a impossibilidade de traçar precisamente as consequências das decisões e ações tomadas, devem ser minoradas pela avaliação continua do produto incorporado para que se promovam várias mudanças incrementais durante o seu ciclo de vida (Rosenfeld, 2013; Balbino et al., 2020).

Os testes contribuíram, de forma ainda preliminar, como um modelo de avaliação da qualidade das fraldas que pode ser bastante útil aos enfermeiros, dando maior noção do impacto do uso das fraldas geriátricas no ambiente hospitalar. Esse entendimento pode resultar em um processo de gestão do produto que realmente funcione. Neste ponto, o uso da escala classificatória de volume miccional empregado a um parâmetro de comparações entre vários tipos de fraldas geriátricas pode ampliar o rol de atributos para atender diferentes necessidades demandada por faixas de comportamento miccional.

As fraldas no mercado de saúde brasileiro são dirigidas aos idosos que têm padrão miccional domiciliar. Então, a variabilidade das fraldas geriátrica, algo de total importância na qualidade. Desse modo, a aplicação da fralda geriátrica aos variados grupos etários, pode estar interferindo nas interpretações dos indicadores de qualidade da assistência no que se refere à pele, pelo desconhecimento do impacto da fralda na incidência de lesões por pressão e umidade por padrão miccional.

O estudo tem como limitação, ser um relato de experiência somente com um lote de fraldas geriátricas de uma marca de fralda. Contudo, com a operacionalização do teste, percebeu-se a necessidade de experimentar volumes diferentes no escore de 3 gotas para determinar o parâmetro que pode molhar a pele. Como também, o cálice graduado em $50 \mathrm{ml}$ se torna inadequado para medidas miccionais mais precisas. 


\section{Considerações Finais}

A umidade é um dos fatores contribuintes para formação de lesões na pele e proliferação de fungos. Assim, as coberturas das fraldas geriátricas devem passar por melhorias para manter o paciente o maior tempo possível com a pele longe da umidade, uma vez que a fralda geriátrica com aplicação de solução com volume de $400 \mathrm{ml}$ e $500 \mathrm{ml}$ foi reprovada na inspeção, toque e presença de umidade, além do teste de peso que correspondia apenas a $1 \frac{1}{4}$ do peso mínimo determinado pelo projetista.

O estudo apontou que existem fortes indícios de que existe a relação da qualidade das fraldas geriátricas com a incidência e prevalência das lesões de pele, algo que deve ser compreendido e explicado pelos pesquisadores para favorecer melhorias nos seus atributos para se adequar ao uso em hospitais.

Enfim, as especificações da fralda geriátrica são insuficientes para avaliação da qualidade do produto. Pontua-se que as diferenças miccionais com uso de uma escala do volume miccional devem ser acrescentadas na embalagem, o que poderá dar transparência aos consumidores, tornando-as possíveis de análises estatísticas de qualidade de produtos industrializados.

\section{Referências}

Back N \& Leal LCM (1992). Uma metodologia de planejamento de testes industriais.

Production, 2(1), 61-9. doi: 10.1590/S0103-65131992000100005.

Balbino, C. M.; Silvino, Z. R.; Joaquim, F., L., Souza, C. J., \& Santos, L. S. (2020). Inovação tecnológica: perspectiva dialógica sob a ótica do Joseph Schumpeter. Research, Society and Development, 9(6), e198963593. doi: 10.33448/rsd-v9i6.3593.

Carpinetti LCR (2012). Gestão da qualidade: conceitos e técnicas. 2.ed. São Paulo: Atlas.

Castilho V \& Gonçalves VLM (2010). Gerenciamento de Recursos Materiais In Kurgant, P (org), Gerenciamento em Enfermagem (pp. 155-67). 2.ed. R.Janeiro, RJ: Guanabara Koogan.

Costa FJC (2011). Mensuração e desenvolvimento de escalas: aplicações em administração. Rio de Janeiro: Ciência Moderna. 
DeFEO JA \& Juran JM (2015). Fundamentos da qualidade. Porto Alegre: Bookman.

Jones TL, Hamilton P \& Murry N (2015). Unfinished nursing care, missed care, and implicitly rationed care: State of the science review. International journal of nursing studies, 52(6), 1121-37. doi: 10.1016/j.ijnurstu.2015.02.012.

Lejcuś, K, Śpitalniak, M., \& Dąbrowska, J. (2018). Swelling Behaviour of Superabsorbent Polymers for Soil Amendment under Different Loads. Polymers, 10(3), 271. doi: 10.3390/polym 10030271 .

Locks MO \& Santos SMA (2016). Uso de Fralda Geriátrica em Hospitais: Solução ou Problema? Rev Estima, 13(1), 27-34. doi: 10.5327/Z1806-3144201500010006.

Longaraya AA \& Ensslin L (2014). Uso da MCDA na identificação e mensuração da performance dos critérios para a certificação dos hospitais de ensino no âmbito do SUS. Production, 24(1), 41-56. doi: 10.1590/S0103-65132013005000021.

Mäki-Turja-Rostedt S, Stolt M, Leino-Kilpi H \& Haavisto E (2019). Preventive interventions for pressure ulcers in long-term older people care facilities: A systematic review. Journal of clinical nursing, 28(13-14): 2420-42. doi: 10.1111/jocn.14767.

Mani ZA \& Ibrahim MA. (2017). Intensive care unit nurses' perceptions of the obstacles to the end of life care in Saudi Arabia. Saudi Medical Journal, 38(7): 715-20. doi:.15537/smj.2017.7.18454.

Marconato JC \& Franchetti SMM. (2002). Polímeros superabsorventes e as fraldas descartáveis: um material alternativo para ensino dos polímeros. Química nova na escola, 15(maio): 42-4. http://qnesc.sbq.org.br/online/qnesc15/v15a09.pdf.

Pittman J, Beeson T, Dillon J, Yang Z \& Cuddigan J (2019). Hospital-Acquired Pressure Injuries in Critical and Progressive Care: Avoidable Versus Unavoidable. American journal of critical care, 28(5): 338-50. https://doi.org/10.4037/ajcc2019264. 
Porzsolt F (2015). Clinical Economics and Nursing. Rev Latino-am Enferm, 23(6), 989-90. doi:10.1590/0104-1169.0000.2640.

Ramos EMLS, Almeida SS, Araújo ARA (2013). Controle estatístico da qualidade. Porto Alegre: Bookman.

Riella MC Príncipios de nefrologia e distúrbios Hidroeletrolíticos. 6.ed. Rio de Janeiro: Guanabara Koogan; 2018.

Rosenfeld H (2013). Gestão de Desenvolvimento de Produtos: uma referência para melhoria de processo. São Paulo: Saraiva.

Vandresen L, Pires DEP, Lorenzetti J \& Andrade SR (2018). Classification of patients and nursing staff's sizing: contributions of a management technology. Rev Gaúcha Enferm., 39, e2017-0107. doi:10.1590/1983-1447.2018.2017-0107.

Vasconcelos MMA, Lima EM, Vaz GB, Silva THS (2013). Disfunção do trato urinário inferior: um diagnóstico comum na prática pediátrica. J. Bras. Nefrol, 35(1), 57-64. doi: 10.5935/01012800.20130009.

Zohuriaan J \& Kabiri K (2008). Superabsorbent Polymer Materials: A Review. Iranian Polymer Journal, 17(6), 2008, 451-77.

https://www.researchgate.net/publication/242582443_Superabsorbent_Polymer_Materials_A _Review.

\section{Porcentagem de contribuição de cada autor no manuscrito}

Alexandra de Oliveira Matias Ferreira - 50\%

Zenith Rosa Silvino - 10\%

Carlos Marcelo Balbino -10\%

Marian Izu - 10\%

Cláudio José de Souza - 10\%

Daniela de Oliveira Matias -10\% 